\title{
Acute maternal oxidant exposure causes susceptibility of the fetal brain to inflammation and oxidative stress
}

Feroz Akhtar ${ }^{1}$, Christopher A. Rouse², Gabriel Catano ${ }^{3}$, Marcus Montalvo ${ }^{1}$, Sarah L. Ullevig ${ }^{4}$, Reto Asmis ${ }^{5}$, Kusum Kharbanda ${ }^{6}$ and Shivani K. Maffi ${ }^{*}$

\begin{abstract}
Background: Maternal exposure to environmental stressors poses a risk to fetal development. Oxidative stress (OS), microglia activation, and inflammation are three tightly linked mechanisms that emerge as a causal factor of neurodevelopmental anomalies associated with prenatal ethanol exposure. Antioxidants such as glutathione (GSH) and CuZnSOD are perturbed, and their manipulation provides evidence for neuroprotection. However, the cellular and molecular effects of GSH alteration in utero on fetal microglia activation and inflammation remain elusive.
\end{abstract}

Methods: Ethanol (EtOH) $(2.5 \mathrm{~g} / \mathrm{kg})$ was administered to pregnant mice at gestational days 16-17. One hour prior to ethanol treatment, $\mathrm{N}$-acetylcysteine (NAC) and L-buthionine sulfoximine (BSO) were administered to modulate glutathione (GSH) content in fetal and maternal brain. Twenty-four hours following ethanol exposure, GSH content and OS in brain tissues were analyzed. Cytokines and chemokines were selected based on their association with distinctive microglia phenotype M1-like (IL-1 $\beta$, IFN $\gamma$, IL-6, CCL3, CCL4, CCL-7, CCL9,) or M2-like (TGF- $\beta$, IL-4, IL-10, CCL2, CCL22, CXCL10, Arg1, Chi1, CCR2 and CXCR2) and measured in the brain by qRT-PCR and ELISA. In addition, Western blot and confocal microscopy techniques in conjunction with EOC13.31 cells exposed to similar ethanol-induced oxidative stress and redox conditions were used to determine the underlying mechanism of microglia activation associated with the observed phenotypic changes.

Results: We show that a single episode of mild to moderate OS in the last trimester of gestation causes GSH depletion, increased protein and lipid peroxidation and inflammatory responses inclined towards a M1-like microglial phenotype $(\mathrm{IL}-1 \beta, \mathrm{IFN}-\gamma)$ in fetal brain tissue observed at 6-24 h post exposure. Maternal brain is resistant to many of these marked changes. Using EOC 13.31 cells, we show that GSH homeostasis in microglia is crucial to restore its anti-inflammatory state and modulate inflammation. Microglia under oxidative stress maintain a predominantly M1 activation state. Additionally, GSH depletion prevents the appearance of the M2-like phenotype, while enhancing morphological changes associated with a M1-like phenotype. This observation is also validated by an increased expression of inflammatory signatures (IL-1 $\beta$, IFN- $\gamma$, IL-6, CCL9, CXCR2). In contrast, conserving intracellular GSH concentrations eliminates OS which precludes the nuclear translocation and more importantly the phosphorylation of the NFkB p105 subunit. These cells show significantly more pronounced elongations, ramifications, and the enhanced expression of M2-like microglial phenotype markers (IL-10, IL-4, TGF- $\beta$, CXCL10, CCL22, Chi, Arg, and CCR2).

\footnotetext{
*Correspondence: shivani.maffi@utrgv.edu

${ }^{1}$ School of Medicine, Department of Biomedical Sciences, Regional Academic Health Center, University of Texas Rio Grande Valley, 1204 W. Schunior, Edinburg 78241, TX, USA

Full list of author information is available at the end of the article
} 
(Continued from previous page)

Conclusions: Taken together, our data show that maintaining GSH homeostasis is not only important for quenching OS in the developing fetal brain, but equally critical to enhance M2 like microglia phenotype, thus suppressing inflammatory responses elicited by environmental stressors.

Keywords: Glutathione, Inflammation, Microglia, Oxidative stress, NF-kB, Fetal alcohol syndrome

\section{Background}

Clinical and preclinical evidence indicates that maternal oxidative stress [1-3] and immune activation are a major source of non-genomic alterations that impair fetal neurodevelopment [4, 5]. Anomalies arise because fetal brain growth and plasticity is strongly influenced by the local cellular metabolic milieu and is highly susceptible to neurochemical perturbations caused by prenatal factors, such as inflammation and environmental stressors [6-8]. Ethanol is a common prenatal environmental stressor known to cause neurocognitive deficits and behavioral abnormalities, the broad effects of which are clinically categorized as Fetal Alcohol Spectrum Disorder (FASD) [2]. Irreversible fetal brain damage occurs due to the loss of glia and neurons in various brain compartments [9-11], the degree of injury being directly dependent on dose, duration of ethanol exposure, and gestational age of the unborn fetus [9, 12]. Additionally, neurons are particularly sensitive to the effects of ethanol during the period of synaptogenesis, also known as the brain growth spurt period, generally observed in the second trimester in rodents and during the last trimester of gestation in humans [9]. The molecular mechanisms underlying FASD are yet to be fully understood; however, there is evidence that increased oxidative stress [13], diminished antioxidant enzymes [1, 14], and more recent reports suggest neuro-immune activation and inflammation [11, 12, 15, 16] all contribute significantly towards ethanol-induced neurotoxicity.

Microglia are the resident immune cells of the central nervous system (CNS). Activation and polarization of microglia is regulated by both endogenous and exogenous factors [17]. Animal models of adult ethanol consumption further indicate that the damaging effects on the brain are due to priming and activation of microglia [18-20], leading to sustained inflammation which is in turn driven by a surge of pro-inflammatory cytokines, such as TNF- $\alpha$, IL-1 $\beta$, and IL-6 [21-24]. Depending on molecular signals received by the microglia receptors, activated microglia acquire either a "cytotoxic M1" or an "alternatively activated M2" neuroprotective phenotype [25]. M1-like phenotype is associated with reduced neurogenesis and deterioration of the neurotrophic system due to the release of pro-inflammatory mediators such as IFN- $\gamma$, IL-1 $\beta$, IL- 6 , and TNF- $\alpha[26,27]$. On the other hand, a M2-like phenotype triggers an array of neuroprotective chemokines. Moreover, microglia's conversion into either classical or alternative phenotype appears to be controlled by two transcriptional factors: nuclear factor kappa-light-chain-enhancer of activated B cells $(\mathrm{NF} \kappa \mathrm{B})$ and nuclear factor (erythroid-derived2)-like 2 (Nrf-2), both extremely sensitive to oxidative stress and redox signaling [28]. While ROS signaling molecules augment p65/p50 dimer formation that subsequently leads to NFkB-dependent transcription of inflammatory cytokines/chemokines associated with a M1-like phenotype, Nrf-2 protects against oxidative damage [29] by activating genes involved in the synthesis of antioxidant enzymes and may likely be a critical regulator of the M2-like phenotype [28]. Despite this knowledge, the synergy of these two transcription factors in modulating microglia function during fetal development and under redox dysfunction remains poorly understood.

In utero ethanol exposure directly activates microglia through TLR2 and TLR4, triggering both oxidative stress and inflammatory cytokine production [11]. Therefore, intracellular factors such as antioxidants (GSH) are likely to influence microglia function [28]. However, it is unclear how and to what extent changes in maternal redox homeostasis impact gestational immune environment and its ultimate influence on microglia activation in the developing fetal brain. We hypothesized that even a single episode of endogenous glutathione dysregulation in utero around mid-gestation is sufficient to cause oxidative stress and inflammatory imprint in the fetal brain. Therefore, in this study, we utilized an in vivo and in vitro model of a short-term shift in oxidative-redox balance to determine the corresponding effect on fetal immune response associated with microglia M1/M2 phenotypic shift.

\section{Methods}

In vivo experimental protocol

\section{Animal model}

Timed pregnant C57 B6 mice were obtained directly from Harlan Laboratories (Indianapolis, IN) at or around gestational day 11. These mice were housed and acclimatized in the 59th Clinical Research Division (San Antonio, TX) animal facility until the day of the experiment. All parameters mentioned below were evaluated in fetal mice of either sex obtained at gestational days 17-18. Seven dams (maternal mice) were placed in each 
of the following treatment groups: control, $\mathrm{EtOH}$ only, $\mathrm{NAC}$ only, $\mathrm{NAC} / \mathrm{EtOH}, \mathrm{BSO}$, and $\mathrm{BSO} / \mathrm{EtOH}$ totaling 42 total dams.

\section{Treatment}

Ethanol, diluted in $20 \%$ saline, was administered to dams subcutaneously at a dose of $2.5 \mathrm{~g} / \mathrm{kg}$. BSO, $1.5 \mathrm{~g} / \mathrm{kg}$, and NAC, $4 \mathrm{mg} /$ dose, intraperitoneal treatments were administered $1 \mathrm{~h}$ prior to alcohol treatment. Twenty-four hours following ethanol exposure, the maternal dams were humanely euthanized via cervical dislocation and the fetuses were harvested. For RNA and protein expression experiments, maternal and fetal brain tissues were flash frozen in liquid nitrogen and stored at $-80^{\circ} \mathrm{C}$ until used. A description of the treatment regimen, dosing, and route of administration is provided in Fig. 1.

\section{In vitro microglia culture protocol \\ Cell culture}

Murine microglia cell line (EOC13.31) was obtained from the American Type Culture Collection (ATCC; Manassas, VA, USA). Cells were maintained in Dulbecco's modified Eagle's medium with $4 \mathrm{mM}$ L-glutamine adjusted to contain $1.5 \mathrm{~g} / \mathrm{l}$ sodium bicarbonate and $4.5 \mathrm{~g} / \mathrm{l}$ glucose, $70 \%$; fetal bovine serum, $10 \%$; and LADMAC conditioned media (produced from the LADMAC cell line (CRL-2420), 20\%, and kept at $37{ }^{\circ} \mathrm{C}$ in a $5 \%$
$\mathrm{CO} 2$ incubator. The culture medium was regularly replenished at 3-4-day interval.

\section{Cell treatment}

Microglia were seeded at the density of $2 \times 10^{5}$ cells $/ \mathrm{mL}$ in either 60 or $100 \mathrm{~mm}$ dishes and were pretreated overnight $(\sim 18-20 \mathrm{~h})$ with PBS (control), NAC $(500 \mu \mathrm{M})$, and BSO $(200 \mu \mathrm{M})$ diluted in PBS [10]. One set from each treated group was further exposed to $22 \mathrm{mM}(\sim 1 \mathrm{mg} / \mathrm{ml})$ ethanol for $6 \mathrm{~h}$. A small ethanol-filled beaker was placed in the incubator to maintain optimum ethanol concentration in the culture media at all times [10].

\section{Total glutathione levels in brain lysates}

Brain extracts were subjected to HPLC analysis for the determination of GSH levels as detailed in [30] and [31] with slight modification. Briefly, brain lysates were prepared in Tris-EDTA buffer + protease inhibitor cocktail (P8340, Sigma Aldrich) to yield a concentration of 30- $\mu$ g protein/ Eppendorf tube. To alkylate the free thiol groups, $100 \mathrm{mM}$ of freshly prepared $\mathrm{N}$-ethylmaleimide (NEM) was added to the samples. Proteins were precipitated with $40 \mu \mathrm{l}$ cold $18 \%$ perchloric acid (PCA). One hundred fifty-microliter aliquots of the supernatant obtained were neutralized with $2 \mathrm{M} \mathrm{KPi}$ and reduced with dithiothreitol (DTT) (final concentration $6.9 \mathrm{mM}$ ). Each sample was diluted with $500 \mu \mathrm{l}$ of $0.1 \mathrm{M} \mathrm{KPi}$ followed by o-phthalaldehyde (final concentration $11.2 \mathrm{mM}$, DTT $<2.4 \mathrm{mM}$ ) to obtain glutathione

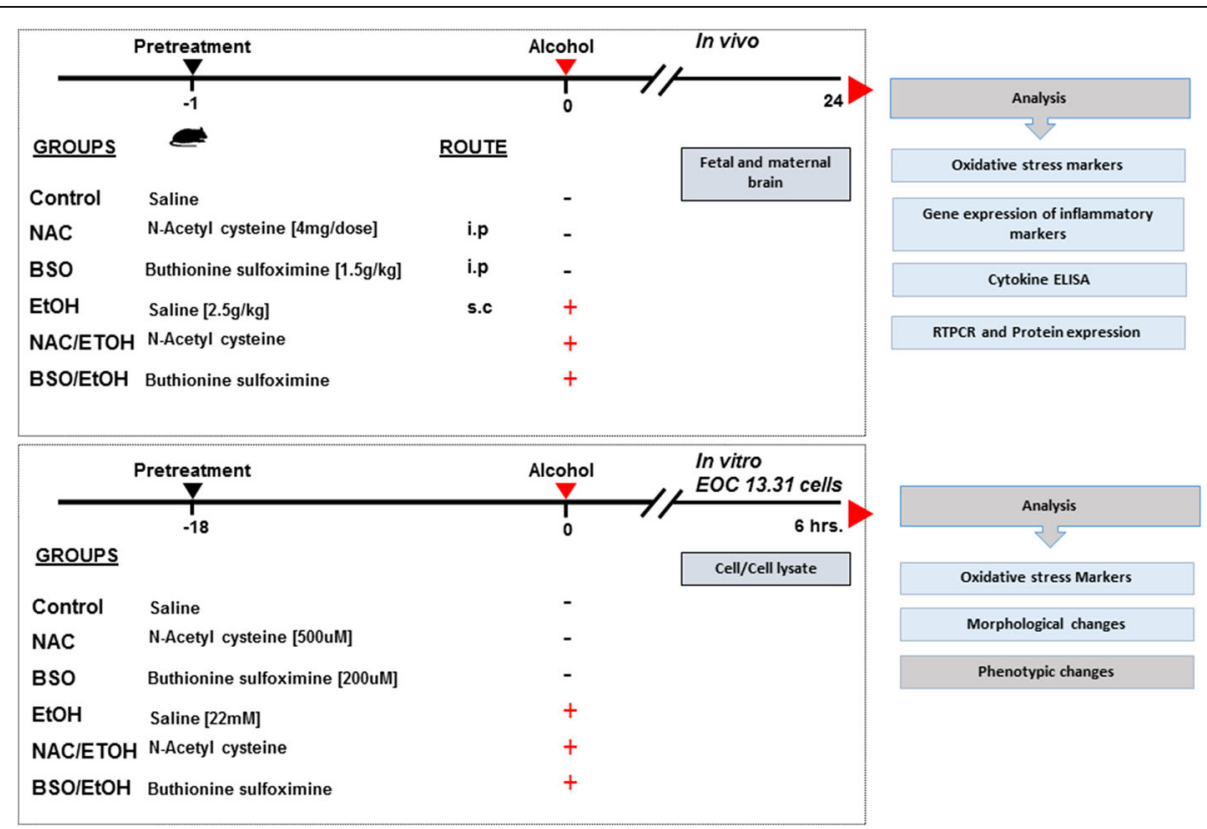

Fig. 1 Overview of the experimental design. Top panel in in vivo studies, pregnant C57 B6 mice were divided into the following groups: Control, $\mathrm{NAC}, \mathrm{BSO}, \mathrm{EtOH}, \mathrm{EtOH} / \mathrm{NAC}$, and BSO/EtOH. For combined treatments, NAC or BSO was administered $1 \mathrm{~h}$ prior to ethanol exposure. Maternal and fetal brain samples were used for further experiments. Bottom panel In vitro model using EOC13.31 cells (microglia cell line) were classified similar to the above 6 groups. Microglia was exposed with NAC or BSO for $18 \mathrm{~h}$ followed by 6 -h ethanol treatment. Live cells and cell lysates were processed further for analysis of oxidative stress, inflammatory, and morphological changes 
derivatives, which were subsequently separated by reverse phase HPLC. HPLC analysis was performed on a Jasco HPLC system equipped with a spectrofluorometer (FP-920, Jasco Inc.) set to an excitation wavelength of $340 \mathrm{~nm}$ and an emission wavelength of $420 \mathrm{~nm}$. Glutathione was separated isocratically on a Brownlee 3-cm C18 ODS guard column $(5 \mu \mathrm{m})$ and a Brownlee 22-cm C18 ODS analytical column $(5 \mu \mathrm{m})$ with $21 \mathrm{mM}$ propionate buffer (in $35 \mathrm{mM} \mathrm{NaPi}, \mathrm{pH}$ 6.5)/acetonitrile (95/5 by volume) at a flow rate of $1.2 \mathrm{ml} / \mathrm{min}$.

\section{Protein carbonyl content}

The reactive carbonyl contents in brain lysates were measured by the widely applied 2, 4-dinitrophenylhydrazine (DNPH) procedure [32]. Proteins $(1-1.5 \mathrm{mg} / \mathrm{ml})$ were separated into two 200- $\mu$ l aliquots (i.e., a test sample and a blank sample). One milliliter of $10 \mathrm{mM}$ DNPH in $2.5 \mathrm{M}$ $\mathrm{HCl}$ was added to the test sample fraction while $1.0 \mathrm{ml}$ of $2.5 \mathrm{M} \mathrm{HCl}$ alone was added to the blank sample fraction. Both fractions were then incubated in the dark at room temperature for $15 \mathrm{~min}$. The samples were precipitated with $1.0 \mathrm{ml}$ of $20 \%$ trichloroacetic (TCA) on ice for $5 \mathrm{~min}$ followed by centrifugation at $10,000 \mathrm{~g}$ for $10 \mathrm{~min}$. Subsequently, the tubes were treated with a 10\% TCA wash, followed by washing in ethanol:ethyl acetate $(1: 1, v / v$, four times). The final pellets were dissolved in $500 \mu \mathrm{l}$ of $6 \mathrm{M}$ guanidine- $\mathrm{HCl}$ in the presence of $20 \mathrm{mM}$ phosphate buffer:trifluoroacetic acid ( $\mathrm{pH}$ 2.3) and left vortexing for $30 \mathrm{~min}$ at $50{ }^{\circ} \mathrm{C}$. The reactive carbonyl content was calculated from its peak absorption at $370 \mathrm{~nm}$ using a molar absorption coefficient $(\varepsilon)$ of $22,000 \mathrm{M}^{-1} \mathrm{~cm}^{-1}$. Reactive carbonyl content $(\mu \mathrm{mol} / \mathrm{l})$ was calculated using BeerLambert equation: Abs380nm (test-blank) $\times 10^{6} / \varepsilon$. The final carbonyl content in the protein was expressed as $\mu \mathrm{mol} / \mathrm{mg}$ protein.

\section{Reverse transcriptase polymerase chain reaction (RT-PCR)} Total RNA was extracted from fetal brains or microglia cells using RNeasy mini kit (Qiagen, Valencia, CA) following the manufacturer's instructions (Catalog 74104). Quantification and analysis of nucleic acid purity were performed with spectrophotometry (NanoDrop Technologies, Wilmington, DE), and $1 \mu \mathrm{g}$ of each sample was reverse transcribed with Moloney murine leukemia virus reverse transcriptase (Superscript II First-Strand Synthesis System for RT-PCR, Invitrogen) in a $20 \mu \mathrm{l}$ of reaction mixture using oligo ( $\mathrm{dT}$ ) primer. Gene expression was measured using real-time PCR. The following primers and FAM-labeled probes from Applied Biosystems Inventoried Assays were used: transforming growth factor beta 1 (TGF- $\beta 1$, cat\# Mm01178820_m1), interleukin-4 (IL-4, cat\# Mm00445259_m1), interleukin-10 (IL-10,cat\#

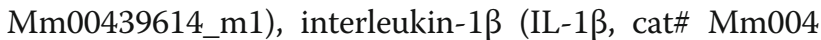
34228_m1), interferon $\gamma$ (IFN- $\gamma$, cat\# Mm01168134_m1), interleukin-6 (IL-6, cat\# Mm00446190_m1), chemokine (C-C motif) ligand 2 (CCL2, cat \#Mm00441242_m1), chemokine (C-C motif) ligand 3 (CCL3, cat \# Mm00 441259_g1), chemokine (C-C motif) ligand 4 (CCL4, cat \# Mm00443111_m1), chemokine (C-C motif) ligand 7 (CCL-7, cat \# Mm00443113_m1), chemokine (C-C motif) ligand 9 (CCL9, cat \# Mm00441260_m1), chemokine (C-C motif) ligand 22 (CCL22, cat \# Mm004 36439_m1), C-X-C motif chemokine 10 (CXC 10,cat \# Mm00445235_m1), C-C chemokine receptor type 2 (CCR2, cat \# Mm99999051_gH), chemokine (C-X-C motif) receptor 2 (CXCR2, cat \# Mm99999117_s1), arginase 1 (Arg-1, cat \# Mm00475988_m1), Chitinase 3-like (CHI3, cat \# Mm00657889_mH), and 18S rRNA (cat \# Mm03928990_g1). Expression of the target genes was determined by qRT-PCR using Gene-specific TaqMan Assay Reagents and TaqMan Gene Expression Assay products on a 7900 HT Fast Real time PCR system (Applied Biosystems, Foster City, CA, USA). Real time PCR was conducted using a 384-well plate (Micro- Amp Fast Optical 96-well Reaction plates and MicroAmp Optical Adhesive Film, both from Applied Biosystems). Reactions were performed in triplicate. Each $10 \mu \mathrm{l}$ reaction contained $0.5 \mu \mathrm{L} 20 \times$ TaqMan gene expression assay, $5 \mu \mathrm{l} 2 \times$ TaqMan universal Master Mix, and $4.5 \mu \mathrm{l}$ of cDNA template. Following one initial step of $95{ }^{\circ} \mathrm{C}$ for $20 \mathrm{~s}$, the cycling parameters were $95^{\circ} \mathrm{C}$ for $1 \mathrm{~s}, 60{ }^{\circ} \mathrm{C}$ for $20 \mathrm{~s}$, and 40 cycles; the data were analyzed using Sequence Detection Systems software (Applied Biosystems), and the cycle number at the linear amplification threshold $(\mathrm{Ct})$ of the endogenous control (18S ribosomal RNA, Applied Biosystems) gene and the target gene was recorded. Relative gene expression (the amount of target, normalized to the endogenous control gene) was calculated using the comparative $\mathrm{Ct}$ method formula $2^{-\Delta \Delta \mathrm{Ct}}$. All PCR data are reported as mean \pm SEM relative expression values.

\section{Western blotting}

Lysates were prepared from brain samples or microglia (EOC13.31) cell lines in chilled RIPA buffer (25 mM Tris-HCL pH 7.6, 150 mM NaCl, 1\% NP-40, 1\% sodium deoxycholate, $0.1 \%$ SDS; Thermo Scientific, Rockford, IL) containing protease inhibitor (complete Mini, EDTA-free Protease inhibitor cocktail tablets, Roche Diagnostics, Indianapolis, IN). Samples were homogenized (3 cycles of $5 \mathrm{~s}$ each) using a cordless pellet pestle motor (Kontes, Fisher Scientific, Pittsburgh, PA) and allowed to lyse for $30 \mathrm{~min}$ on ice, followed by centrifugation at $13.2(\times 10,000) \mathrm{rpm}$ for $15 \mathrm{~min}$ (Eppendorf Centrifuge 5415 D, Eppendorf North America, Hauppauge, NY), and the cleared supernatant was collected and stored at $-20{ }^{\circ} \mathrm{C}$. Nuclear and cytoplasmic proteins were extracted separately using the NE-PER Nuclear and 
cytoplasmic Extraction Reagents (Thermo scientific). Protein concentrations were determined using BCA kit (Pierce BCA Protein Assay Kit; Thermo Scientific, Rockford, IL). Fifteen to $20 \mu \mathrm{g}$ of protein samples were separated on a SDS polyacrylamide gel (10-15\%) and subsequently electrophoretically transferred onto nitrocellulose membranes (Thermoscientific), followed by blocking with nonfat dry milk in TBST $(50 \mathrm{mM}$ Tris-Hcl, $\mathrm{pH}$ 7.4, $150 \mathrm{mM} \mathrm{NaCl}, 0.2 \%$ Tween 20). The membranes were incubated overnight with any of the mentioned primary antibodies: rabbit anti mouse Nrf-2 (1:1000; Thermo Fisher Scientific, cat\# PA5-27882), rabbit anti mouse 4HNE (1:1000; Abcam, cat\# ab46545), rabbit anti mouse Phospho-NF-kB p105 (1:1000; Cell Signaling, cat\#4806), rabbit anti mouse NF-кB p105 p105/p50 (1:1000; Cell Signaling, cat\#13586), rabbit mAb NF-kB p65 (1:1000; Cell Signaling, Cat\#8242), Phospho-NF-кB p65 (1:1000; Cell Signaling, Cat\#3033), and anti-mouse $ß$-actin(1:10,000; Sigma cat\#A5441), followed by HRP labeled goat anti mouse or goat anti rabbit or goat anti sheep (1:1000; Santa Cruz). Protein bands were detected using a chemiluminescence (ECL kit) method (Pierce) and visualized on $\mathrm{x}$-ray film (Kodak).

\section{Cytokine ELISA}

The concentration of the following cytokines in the brain lysates were determined using ELISA kits according to the manufacturer's instructions: IL-10 (ThermoFisher Scientific, cat\# KMC0101), IL-6 (ThermoFisher Scientific, cat\# KMC0061), IFN- $\gamma$ (ThermoFisher Scientific, cat\# KMC4021), TNF- $\alpha$ (ThermoFisher Scientific, cat\# KMC3011), and IL1 $\beta$ (ThermoFisher Scientific, cat\# KMC0011).

\section{Cell viability}

Microglia were seeded at a density of 5000 cells/well in a 96-well plate, and the cell viability was determined using MTS assay (Promega; cat\#G3580). Briefly, at the end of treatment regimen, $100 \mu \mathrm{l}$ of media was removed and $20 \mu \mathrm{l}$ of Cell Titer 96Aq reagent was added. The plates were incubated for $1.5 \mathrm{~h}$ at $37{ }^{\circ} \mathrm{C}$ to allow the MTS tetrazolium compound to convert into a colored soluble formazan. Absorbance was recorded at $490 \mathrm{~nm}$ using a microplate reader (Spectra Max, Molecular Devices).

\section{Cellular glutathione content}

GSH levels were determined as described by Kamencic et al. with slight modification [33]. Cells were cultured in 96-well plates as described above and subjected to various treatments, after which wells were washed with PBS and incubated with $40 \mu \mathrm{M}$ monochlorobimane (MCB) in the dark for $30 \mathrm{~min}$ at $37^{\circ} \mathrm{C}$, followed by two further washes with PBS. Fluorescence intensity was measured using a spectrofluorophotometer microplate reader (SpectraMax, MS Molecular Devices), with excitation and emission wavelengths of 405 and $510 \mathrm{~nm}$, respectively. Samples were assayed in triplicates.

\section{Reactive oxygen species (ROS) detection}

ROS generation was measured by labeling cells with CellROX Deep Red Reagent following the manufacturer's instructions (Molecular Probes, Life Technologies). CellROX deep red is a cell permeable non-fluorescent dye that is oxidized by cytoplasmic free radicals to emit fluorescence. Microglia were grown on 35-mm glass bottom dishes (MatTeK Corps). After various treatments, cells were washed twice and loaded with $5 \mu \mathrm{M}$ of the fluorescent probe for $1 \mathrm{~h}$ at $37^{\circ} \mathrm{C}$. Cells were washed three times with PBS and imaged immediately. Multiple random images were captured using an Olympus FV1000 confocal microscope equipped with a $\mathrm{HeNe} 635 \mathrm{~nm}$ laser, 20x objective, NA 0.75 with an electronic zoom of 1.2. Laser intensity, scan speed, and other settings were attenuated to minimize phototoxicity and photobleaching. In addition, for reproducibility and comparison purposes, all microscope settings were kept identical across all treatment groups.

\section{Analysis of cell morphology}

Differential Interference Contrast (DIC) images of microglia grown on $35-\mathrm{mm}$ glass bottom dishes were captured using a Fluoview FV 1000 Olympus confocal microscope equipped with $\mathrm{HeNe}$ laser, 20x objective, NA 0.75 . Morphological analysis was conducted as described by McWhorter et al. [34]. Briefly, NIH ImageJ software was used to trace and measure long and short axis of each cell manually. The long axis was defined as the longest length of the cell, and the short axis was defined as the length across the nucleus in a direction perpendicular to the long axis. The ratio of the two axes was determined and considered as the elongation factor.

\section{Immunofluorescence microscopy}

After treatment, cells were fixed in 4\% paraformaldehyde for $20 \mathrm{~min}$, then permeabilized with $0.2 \%$ saponin in $10 \%$ FBS-PBS for $20 \mathrm{~min}$ at room temperature, and stained with goat anti Arginase 1 (1:200; Santa Cruz, cat\# SC18355), goat anti IL-1 $\beta$ (1:300; Abcam, cat \#ab195991), and a secondary antibody conjugated with Alexa Fluor 647 or Alexa Fluor 488 (1:200, Abcam). Cells were mounted using VectaShield mounting medium containing DAPI (Fisher Scientific), and images were visualized using an Olympus FV1000 confocal microscope with a $60 \times$ PlanApoN objective, NA 1.42 using inbuilt 405-nm diode, 488-nm argon, and 635-nm diode laser settings. Images were captured sequentially with a scan speed of $12.5 \mu \mathrm{s} /$ pixel. Acquisition settings were offset to minimize photobleaching and also set 
using appropriate iso-controls. In addition, microscope settings were kept identical for each treatment group.

\section{Statistics}

Statistical analyses were performed using either Stata 11(College Station, TX) or sigma plot 12.0. Student's T test or one-way analysis of variance or StudentNewman-Keuls Method were applied for pairwise multiple comparisons. All data are presented as mean \pm SEM. Compounded.

\section{Results}

Maternal exposure to a single episode of GSH imbalance increases ethanol-induced oxidative damage in the fetal brain

The susceptibility of the fetal brain to oxidative stress produced by ethanol is likely to be augmented by its underdeveloped antioxidant machinery. Therefore, as a first step, the contribution of maternal antioxidant status to oxidative damage in the fetal brain was evaluated by measuring the levels of reduced glutathione and protein and lipid peroxidation products (carbonyls and 4-HNE adducts) at $24 \mathrm{~h}$, following a single episode of moderate ethanol exposure in dams pretreated with two GSH modulators, NAC or BSO. NAC is a weak ROS scavenger that supplies cysteine for GSH synthesis, while BSO is an irreversible inhibitor of $\gamma$-glutamylcysteine, the rate-limiting step in GSH synthesis, and is extensively used to deplete GSH levels. Ethanol treatment alone did not produce any significant change in GSH levels in the fetal brain. Compared to control or ethanol-treated group, pretreatment of dams with BSO alone or with ethanol significantly reduced the GSH content by 79.5 and $83.8 \%$ in the fetal brain $(p<0.005)$. However, no changes in GSH levels were observed in the maternal brains from either treated or control mice $(p<0.08)$ (Fig. 1b). The presence of protein carbonyls and 4-HNE represents the extent of oxidative damage induced by ROS. The decrease in GSH levels following BSO pretreatment corresponded with increased protein and lipid peroxidation products, like 4-HNE, in fetal brain homogenates. Carbonyl content was sharply increased in the fetal brains exposed to BSO (+365\%; $p<0.005), \mathrm{BSO}+\mathrm{EtOH}(401 \% ; p<0.005)$, and $\mathrm{EtOH}(+$ $295 \%$; $p<0.05$ ) when compared to control (Fig. 2c). Notably, maternal brain was resistant to protein oxidation when exposed to ethanol alone $(p<0.060)$. However, administration of BSO before EtOH exposure significantly enhanced the levels of protein carbonyls $(p<0.005)$ in comparison to the control or the ethanol-treated group (Fig. 2d). Ethanol-induced protein oxidation was completely prevented by supplementation with NAC in both fetal and maternal brain (Fig. 2c, d). Furthermore, we probed immunoblots of brain homogenates with antibodies directed against 4-HNE, a cytotoxic breakdown product of fatty acid peroxides, and found levels of 4HNE were noticeably increased in both fetal and maternal brains exposed to $\mathrm{EtOH}, \mathrm{BSO}$, or $\mathrm{BSO}+\mathrm{EtOH}$, when compared with control (Fig. 2e, f) as evidenced by the presence of protein bands of approximately $76,60,50$, and $37 \mathrm{kDa}$. This effect was reversed by pretreatment with NAC similar to carbonyl content in the control group.

\section{Oxidative-redox shift causes divergent immune responses in fetal and maternal brain}

Redox imbalance and oxidative stress are known to elicit transcriptional induction of inflammatory genes [28]. Thus, we initially set to evaluate the effect of ethanol and GSH modulation on selected pro- and anti-inflammatory mRNA expression and also the subsequent changes in cytokine proteins in fetal and maternal brain. To determine changes in gene expression for each treatment regimen, values are expressed as fold change over the corresponding control and analyzed using independent Student's $t$ test. Since differential expression of inflammatory mediators are known to drive microglia to acquire either M1-like or M2-like phenotypes [35], we grouped these markers according to their association with distinctive microglia phenotype M1 (IL-1 $\beta$, IFN- $\gamma$, IL-6, CCL3, CCL4, CCL-7, CCL9,) or M2 phenotype (TGF- $\beta$, IL-4, IL10, CCL2, CCL22, CXCL10, Arg1, Chi1, CCR2, and CXCR2). Exposure to BSO or EtOH alone or their combination upregulated the expression of inflammatory cytokines (IL-1 $\beta$, IFN- $\gamma$, IL-6) in the fetal brain, when compared to control (Fig. 3a), although only IL-1 $\beta$ and IFN- $\gamma$ expression was significantly affected by BSO and EtOH treatments $(p<0.05)$. In contrast, NAC pretreatment significantly downregulated the expression of both, IL- $1 \beta$ and IFN- $\gamma$ in the fetal brain, in the absence and presence of ethanol ( $p<0.05$ and $p<0.005$, respectively). Elevated expression of IL-6 was observed across all the groups, though not statistically significant. The most notable anti-inflammatory M2 cytokine was IL-10: its expression was significantly suppressed in the group treated with $\mathrm{BSO}+\mathrm{EtOH}$ ( $p<0.05$ vs control) (Fig. 3a). Conversely, the expression of M2-associated cytokines was upregulated in NAC or NAC + EtOH-exposed fetuses. Modulation of these inflammatory markers was less evident in the maternal brain. However, IL-6 was clearly upregulated in $\mathrm{NAC}, \mathrm{NAC}+\mathrm{EtOH}, \mathrm{BSO}, \mathrm{BSO}+\mathrm{EtOH}$ groups, and TGF $\beta$ was downregulated in $\mathrm{BSO}+\mathrm{EtOH}$ group $(p<0.05)$. Surprisingly, none of the chemokines measured showed any significant changes in gene expression in either maternal or fetal brain; however, an overall trend of upregulation of M1-associated chemokines (CCL3, CCL4, CCL7, CCL9) and downregulation of M2-associated chemokines (CCL2, CXCL10, and CCL22) was observed in GSH depleted fetal brain. Similarly, NAC pretreatment appeared to inhibit the expression of M1-associated chemokine 

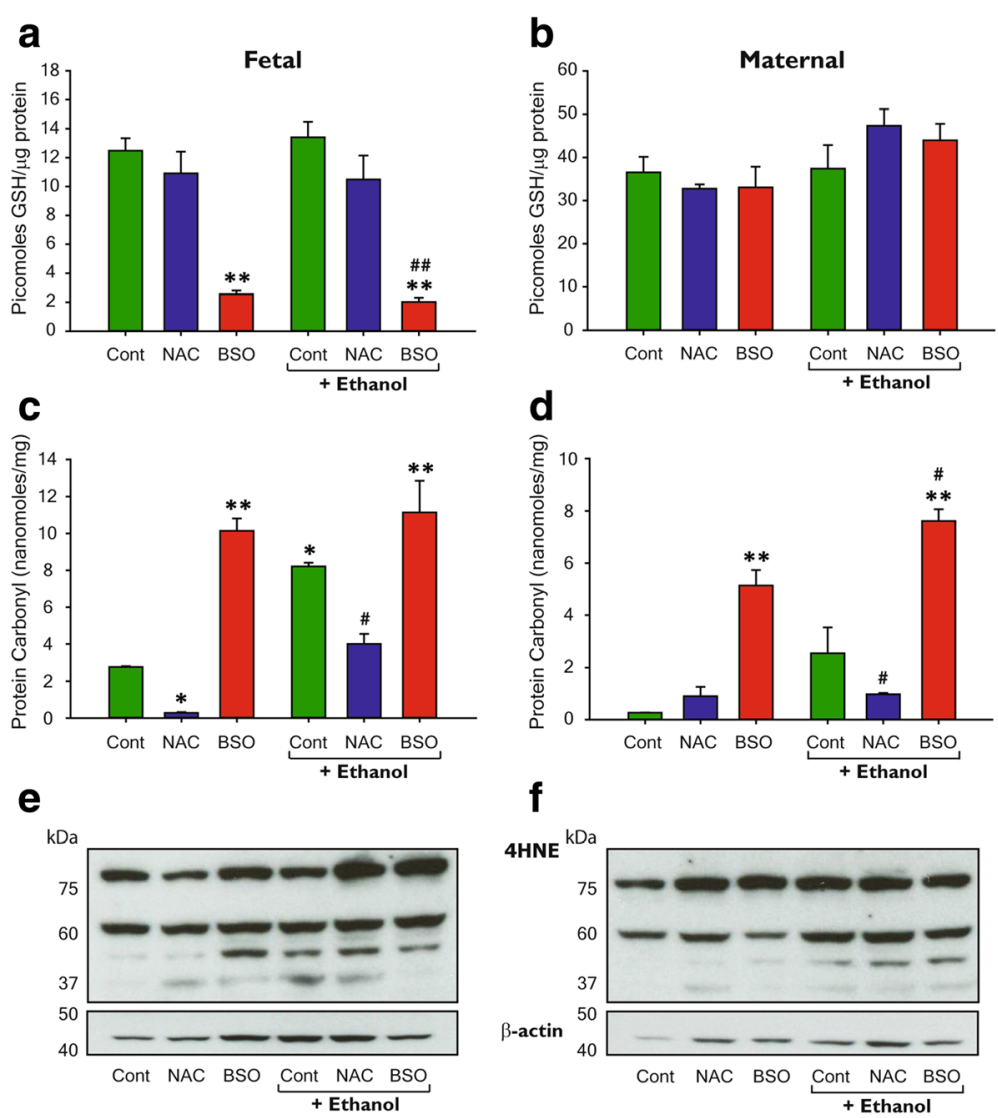

Fig. 2 Ethanol and glutathione depletion generate oxidative stress in fetal and maternal brain. Twenty-four hours after dams were subcutaneously administered ethanol $(2.5 \mathrm{~g} / \mathrm{kg}$ ), following $1 \mathrm{~h}$ intraperitoneal pretreatment with either NAC (4 mg/dose) or BSO (1.5 g/kg), total GSH levels were determined by HPLC in fetal (a) and maternal (b) brain lysates. 2,4-Dinitrophenylhydrazine (DNP) derivatized protein carbonylation levels in lysates were quantified spectrophotometrically in fetal (c) and maternal (d) brains. Expression of 4-Hydroxynonenal adduct formation was used to determine lipid peroxidation in the brain. A representative immunoblot against $4 \mathrm{HNE}$ in fetal (e) and maternal (f) lysates. Values are mean \pm SEM $\left({ }^{*} p \leq 0.05,{ }^{* *} p \leq 0.005\right.$ vs control; $\# p \leq 0.05, \# \# \leq 0.005$ vs ethanol), $(n=6$ per group)

genes (CCL3, CCL4, CCL9) and simultaneously increase the expression of M2-associated chemokines (CCL2, CXCL10, CCL22) (Fig. 3c).

We next tested the expression of phenotype-specific markers Arg1, Chi, CCR2, and CXCR2, which indicate the polarization of microglia towards an M2 phenotype. More specifically, Arg1 was significantly induced in fetuses exposed to NAC $(p<0.001)$, when compared to control; however, ethanol treatment mitigates this response. In contrast, Arg1, Chi1, and CCR2 expression were reduced in fetal brain from $\mathrm{BSO}$ and $\mathrm{BSO}+\mathrm{EtOH}$ groups. With respect to maternal brain, the following significant changes were observed in maternal brain: upregulation of Arg1 following EtOH exposure, upregulation of CCR-2 upon exposure to $\mathrm{BSO}+\mathrm{EtOH}$, and suppression of CXCR-2 and Chi with NAC exposure $(p<0.05)$ (Fig. 4a, b).

Cytokines such as IL-1 $\beta$, TNF- $\alpha$, IL-6, IL-10, and IFN- $\gamma$ also play a key role in neuronal differentiation [36], survival [37], and pathological profile that emerges in later life [38]. Therefore, we next determined protein expression of these cytokines by ELISA in lysates of fetal and maternal brain. Ethanol exposure increased IFN- $\gamma$ $(p<0.05)$, but this effect was mitigated by NAC pretreatment $(p<0.025)$. A M2 phenotypic (IL-10) response was observed in fetal brain from the group with NAC exposed alone $(p<0.025)$. High levels of IL-1 $\beta$ are known to occur in the developing brain, and these levels taper off towards the end of gestation [39]. No significant differences in relative cytokine protein levels were detected in maternal brain lysates between the treatment groups (Fig. 4d).

Nrf-2 activation promotes cell survival despite loss in GSH and increased oS

In order to identify key components of the inflammatory cascade in the fetal brain, we next employed an in vitro model using microglia EOC13.31 cells subjected to similar ethanol-induced oxidative stress with varying redox status, in the presence or absence of NAC or BSO pretreatment. Initially, cell toxicity of various concentrations 

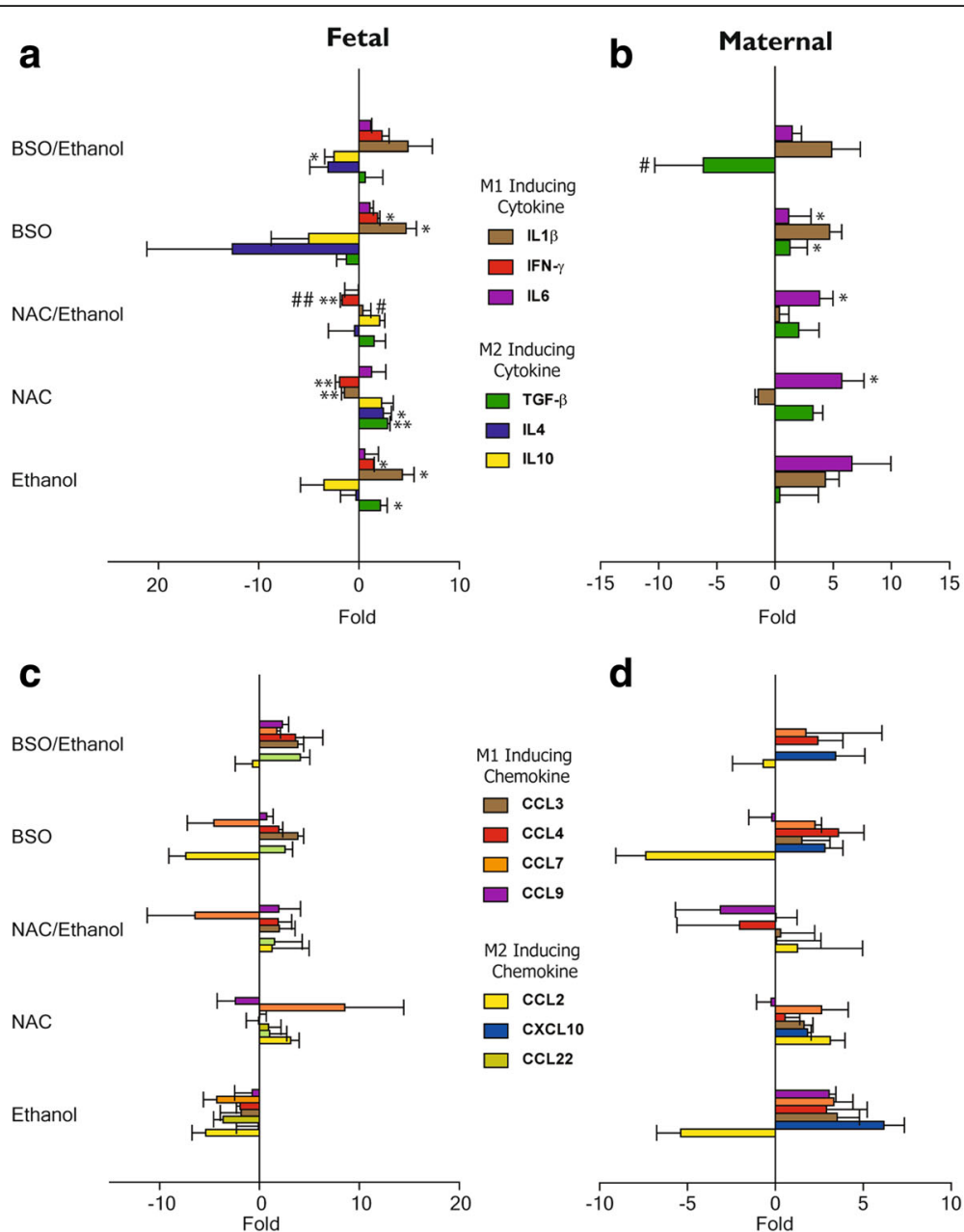

Fig. 3 Effect of Ethanol and glutathione modulation on inflammatory response in fetal and maternal brain. Gestational exposure to ethanol and GSH variation has a direct consequence on fetal cytokine/chemokine RNA expression as determined by qRT-PCR. Levels were normalized to $18 \mathrm{~S}$ rRNA and expressed relative to control. Cytokine and chemokine response in the fetal $(\mathbf{a}, \mathbf{c})$ and maternal $(\mathbf{b}$, $\mathbf{d})$ brain, respectively, were further outlined based on their distinct association with microglia M1 or M2 phenotype. Values are mean \pm SEM. $\left({ }^{*} p \leq 0.05,{ }^{* *} p \leq 0.005\right.$ vs control; \# $p \leq 0.05$, \#\# $p \leq 0.005$ vs ethanol) ( $n=4-6$ per group)

of ethanol was tested using MTS assay which indicated a significant loss in cell viability of microglia at $44 \mathrm{mM}$ ethanol and above (Additional file 1: Figure S1). Therefore, an optimum non-cytotoxic dose of $22 \mathrm{mM}$ ethanol for $6 \mathrm{~h}$ was selected for all subsequent experiments. This dose is physiologically relevant in humans and is reflective of blood alcohol levels reported during $\mathrm{EtOH}$ intoxication [40]. A comparison of oxidative stress levels in microglia between various groups was determined using confocal microscopy (Fig. 5a). Quantitative analysis of the fluorescence intensities by Image J (Fig. 5b) demonstrated an increased ROS generation in microglia exposed to EtOH and BSO alone $(p<0.05)$ or in combination $(p<0.005)$, as compared to control (saline). Interestingly, the fluorescence signal was diminished in cells treated with NAC+ $\mathrm{EtOH}$, as compared to EtOH group $(p<0.05)$. Since oxidative stress rapidly converts reduced GSH into its oxidized GSSG state, thus depleting the total cellular GSH pool, we also measured GSH levels using the fluorochrome monochlorobimane (Fig. 5c). Exposure to EtOH and BSO either alone or in combination significantly reduced intracellular GSH content $(p>0.005)$. Similar to in vivo conditions, intracellular GSH levels were neither significantly altered after overnight NAC pretreatment alone nor perturbed when NAC treatment was followed by EtOH exposure (Fig. 5c). We next examined the expression of Nrf-2, a transcription factor known to trigger expression of several antioxidant enzymes which is an important cellular defense and survival mechanism against oxidative stress, especially following EtOH and BSO exposure. Western blot analysis in Fig. 5 d shows an increase in nuclear accumulation of Nrf-2 expression in microglia pretreated with $\mathrm{BSO}$ and/or exposed to $\mathrm{EtOH}$ that was concurrent with a decline in cytosolic Nrf-2. 

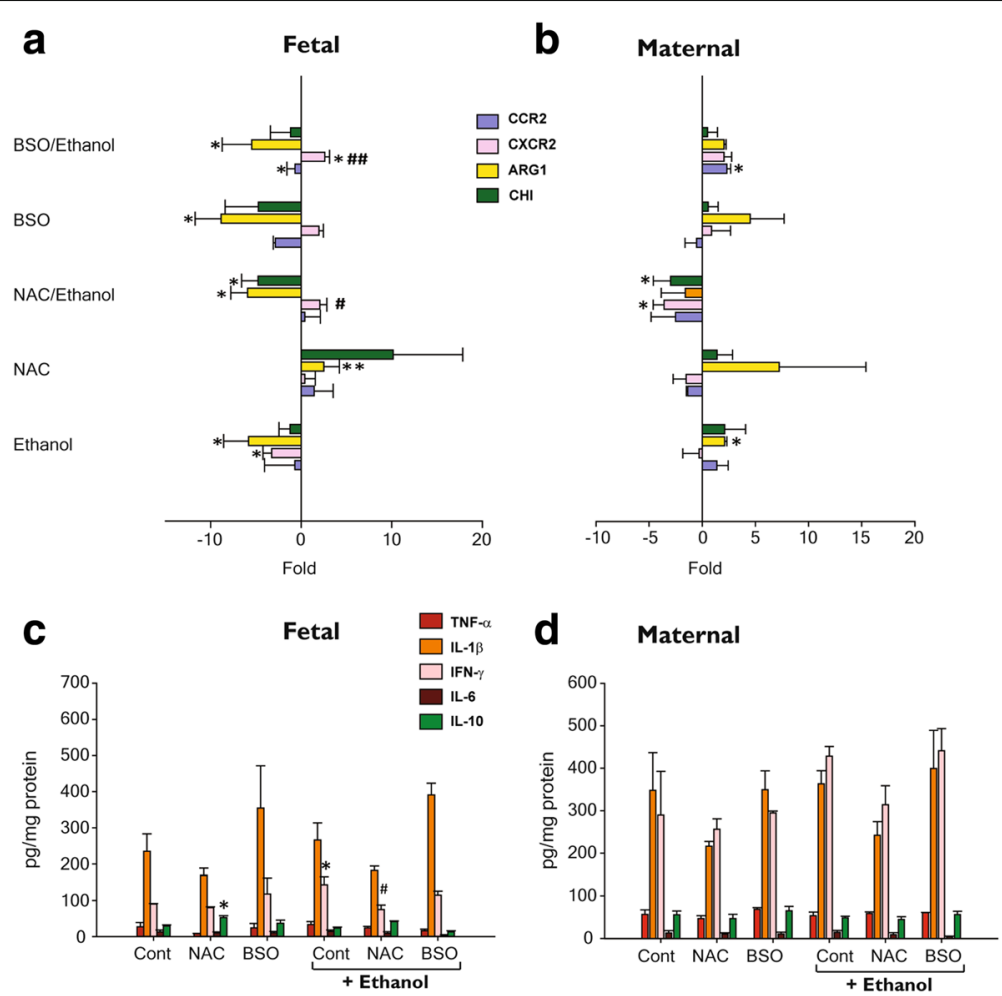

Fig. 4 Effect of oxidative stress on inflammatory markers and protein expression. qRT-PCR analysis of M1 (CXCR2) and M2 (Arg-1,Chi,CCR2) specific markers in the fetal (a) and maternal (b) brain. Expression level of each gene was normalized to 185 rRNA and expressed relative to the control group. Values are \pm SEM. Protein level expression of cytokines in the fetal (c) and maternal (d) brain. Values are mean \pm SEM. $\left({ }^{*} p \leq 0.05\right.$, **p $\leq 0.005$ vs control; $\# p \leq 0.05$, \#\#p $\leq 0.005$ vs ethanol) ( $n=6$ per group)

Thus, it appears that GSH depletion facilitates the accumulation of Nrf-2 in the nucleus. Consistent with this result, expression of CuZnSOD, an antioxidant, was enhanced in GSH depleted cells exposed to EtOH (Additional file 2: Figure S2), which is indicative of a concurrent defense mechanism to quench the free radicals being generated and thus inhibiting ROS-mediated cytotoxicity (Fig. 5e).

\section{Microglia GSH content impacts differential expression of M1 and M2 markers and morphological changes}

Microglia are morphologically dynamic cells whose morphological changes appear to be closely associated with their functional activities. Overall, under normal conditions, microglia grow as a mixed population, consisting predominantly of bipolar rod-like structures, some with extensive ramifications, in addition to a small percentage of rounded cells. Both representative confocal DIC images (Fig. 6a) along with quantification of the morphological observations (Elongation factor (EF)) (Fig. 6b) demonstrate that oxidative stress whether induced by $\mathrm{EtOH}$ or $\mathrm{BSO}$ or their combination had a prominent influence on microglia morphology $p<0.001$. While ethanol exposure caused microglia to predominantly assume a round-amoeboid shape, with fewer cells retaining ramifications, BSO exposure caused almost $85 \%$ of cells to become rounded. Despite noticeable cellular elongations and extensive ramifications, the presence of NAC did not yield any significant difference $(p=0.155)$, as compared to control (Fig. 6b). Intriguingly, NAC pretreatment prior to $\mathrm{EtOH}$ exposure allowed microglia to retain their ramified state, whether compared to control or ethanol group ( $p=0.006$ and 0.008 , respectively). Such obvious morphological changes possibly are interlocked with functional activities; thus, we next examined the mRNA expression of M1/M2-like phenotype signatories driving the microglia polarization states (Fig. 6c). $\mathrm{EtOH}$ or BSO exposure significantly increased the gene expression of IL-1 $\beta$ and IL-6, both M1 pro-inflammatory markers. Conversely, NAC pretreatment significantly downregulated the expression of IL- $1 \beta$ and IL- 6 and, as expected, upregulated IL-10 and CCL2, both markers of M2 polarization. Consistent with this, immunofluorescent microscopy images of microglia exposed to $\mathrm{EtOH}, \mathrm{BSO}$ alone, or their combination showed IL-1 $\beta$ expression and, concurrently, an extremely low expression of Arg1, an M2 marker (Fig. 7). On the other hand, NAC treatment inhibited the expression of M1 markers (Fig. 7 and Additional file 3: Figure S3) while simultaneously enhancing Arg1 expression. Taken together, these 


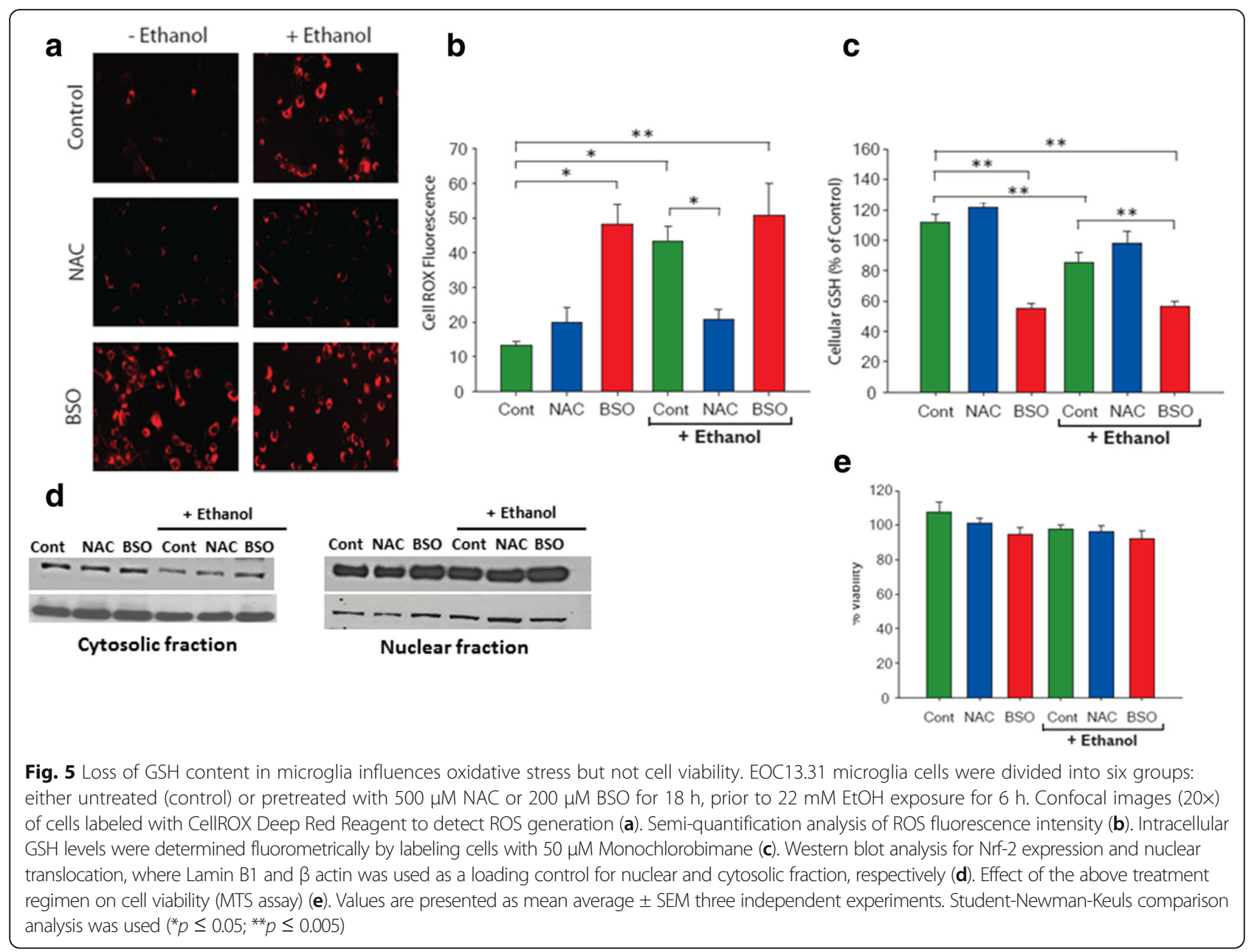

findings demonstrate that ROS and intracellular GSH directly regulate microglia phenotype, expression of inflammatory markers, and the positive relationship between M2 polarization and cell elongation.

\section{Activation of NF-KB contributes to acquisition of pro-inflammatory phenotypes}

Depending on the stimuli and cell type, NF- $\kappa B$ signaling serves a dual function in the brain and is directly associated with inflammation, neuroprotection, neurotransmission, cell death, and cell survival [41-43]. The NF-kB family of transcriptional factors are redox sensitive, dysregulated redox homeostasis may impact NF- $\mathrm{kB}$ activation and subsequent release of pro-inflammatory mediators by microglia. Thus, in order to understand the underlying mechanism associated with our treatment regimens, we explored the activation and expression of NF- $\mathrm{BB}$ subunits: p105, p50, and p65/RelA. Western blotting results show increased processing of p105 into p50 subunit, elevated expression of p65, and nuclear translocation of p50 in $\mathrm{BSO}+\mathrm{EtOH}$-treated cells (Fig. 8a-c), suggesting activation of the classical NF- $\mathrm{kB}$ pathway in vitro in microglia depleted of GSH which is augmented by exposure to ethanol. Moreover, ethanol treatment dampened the phosphorylation of p105 and p65 across all groups. Consistent with this, we also observed upregulated expression of p50 in fetal brain exposed to EtOH or $\mathrm{BSO}$ alone or in combination (Fig. 8d).

\section{Discussion}

Our results reaffirm that oxidative stress $[13,44]$ is a causal factor for several immunological and neurological impairments [45] observed in FASD. More importantly, these results show that maternal antioxidant status has direct consequences on fetal brain development; therefore, even a minor short-term shift in redox-oxidative balance during gestation increases the possibility of fetotoxicity from ethanol exposure. To this end, moderate single doses of ethanol, NAC, and BSO were administered at gestational days 16-17 in mice. Twenty-four hours later, we determined the extent of oxidative stress and immune status in the brains of dams and fetuses using microglia M1/M2 paradigm comparison. We show that $\mathrm{NAC}, \mathrm{BSO}$, and ethanol all had a predominant 


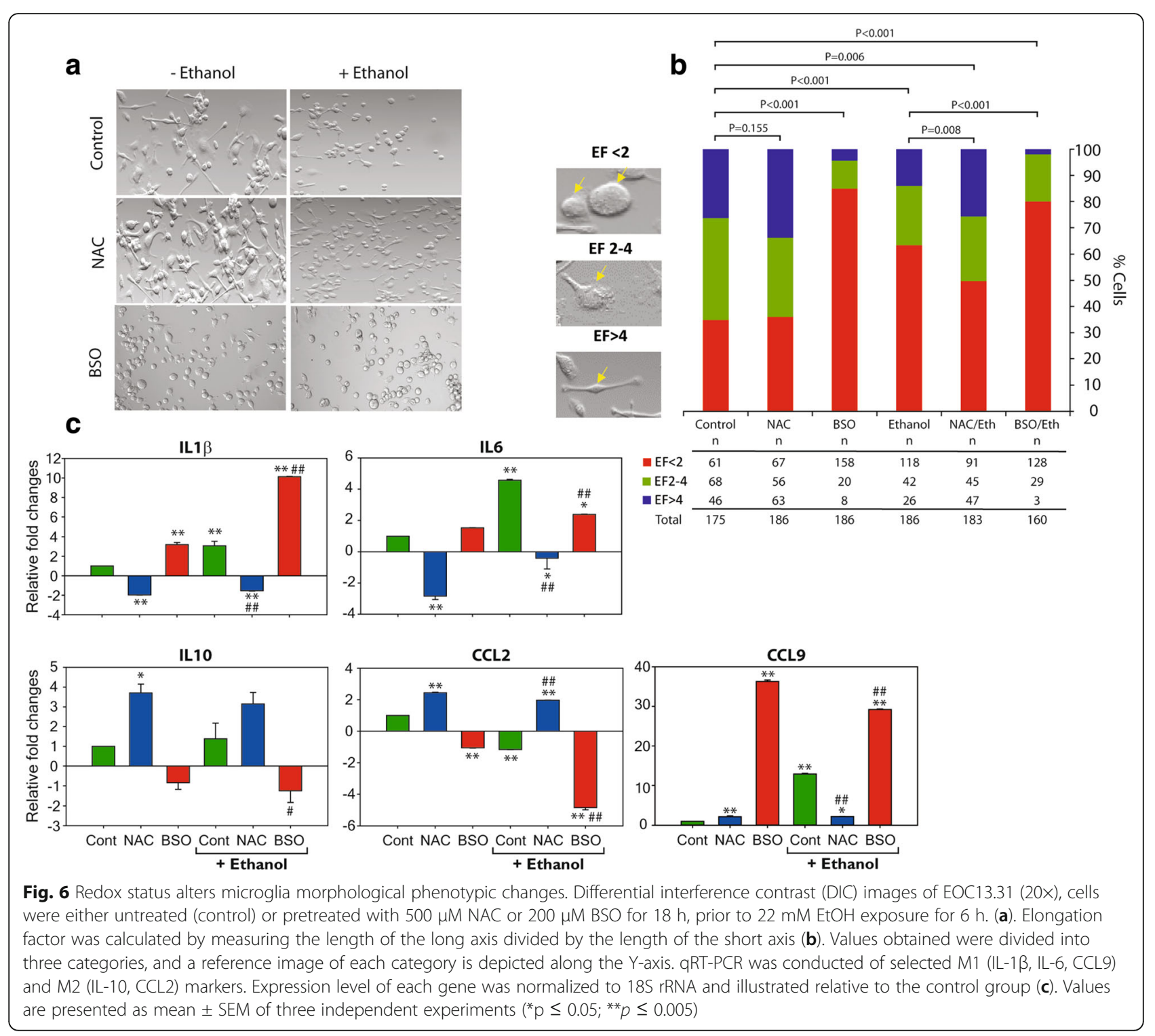

effect on the fetal brain and less on the maternal brain. Pronounced oxidative damage characterized the fetal brain response to redox imbalance alone and sometimes in conjunction with ethanol exposure, as seen by increased oxidation of both protein and lipids, as well as GSH depletion (Fig. 2). Although maternal ethanol consumption is reported to reduce GSH content of the fetal brain $[46,47]$, we observed no such effect. Surprisingly, NAC pretreatment used in this study did not alter GSH levels, unlike restoration of GSH levels by NAC as reported by others [48]. These variations in findings are likely due to difference in administration routes and exposure regimens adopted by us and others.

Our work and evidence from earlier studies show that redox imbalance and oxidative stress are central to induction of inflammatory genes [28]. Moreover, when these factors are combined with ethanol, they appear to leave a persisting inflammatory signature in the offspring $[15,49]$. A number of earlier studies suggest microglia activation as the source of inflammation and tissue damage following ethanol intake. However, microglial "activation" which is highly dependent on cytokines and chemokines present in the surrounding milieu may or may not necessarily lead to an inflammatory response $[12,23,24,50-52]$. Therefore, it is important to distinguish that once activated, microglia can acquire either a detrimental neurotoxic (M1)-like phenotype [11, 49, 53, $54]$ or a neuroprotective (M2)-like phenotype [25, 54]. Low tissue levels of GSH quite often exacerbated by environmental stressors (ethanol exposure) cause microglia activation to trigger the pro-inflammatory response whereas maintaining optimal tissue GSH concentrations not only abate the neurotoxic response of ethanol but also provide cues for switching to an anti-inflammatory 


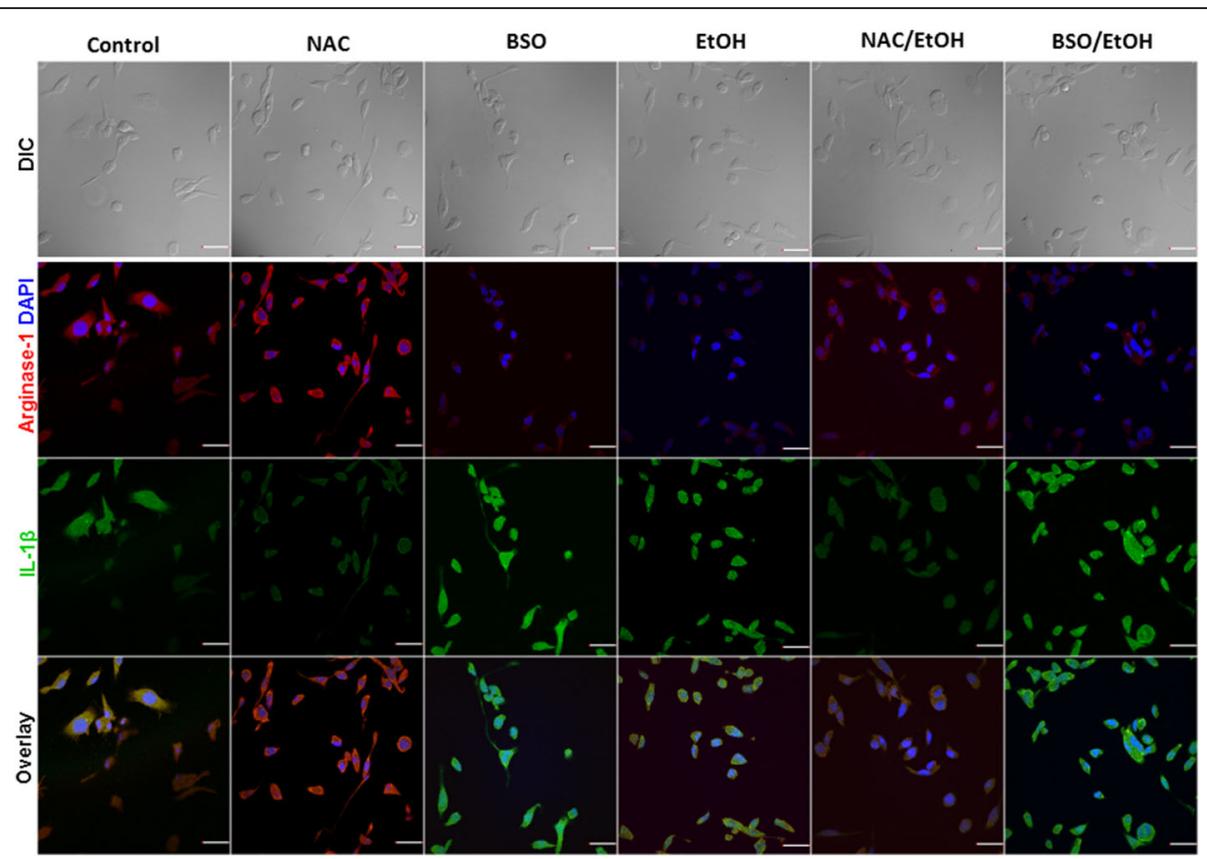

Fig. 7 Oxidative stress and intracellular GSH dictate M1/M2 phenotypic acquisition of microglia. EOC 13.31 cells grown on coverslips were pretreated with either NAC $(500 \mu \mathrm{M})$ or BSO $(200 \mu \mathrm{M})$ for $18 \mathrm{~h}$, followed by either absence or presence of EtOH $(22 \mathrm{mM})$ exposure for 6 h. Immunohistochemistry was performed using antibodies against IL-1 3 (M1), Arginase1 (M2) and subsequently visualized with Alexa Fluor 488 (green) and Alexa Fluor 647 (red) labeled secondary antibodies, respectively. DAPI was used as a nuclear stain. Images were captured with a confocal microscope using a 60x objective. Scale bar $=40 \mu \mathrm{m}$. Images shown are representative from three independent experiments

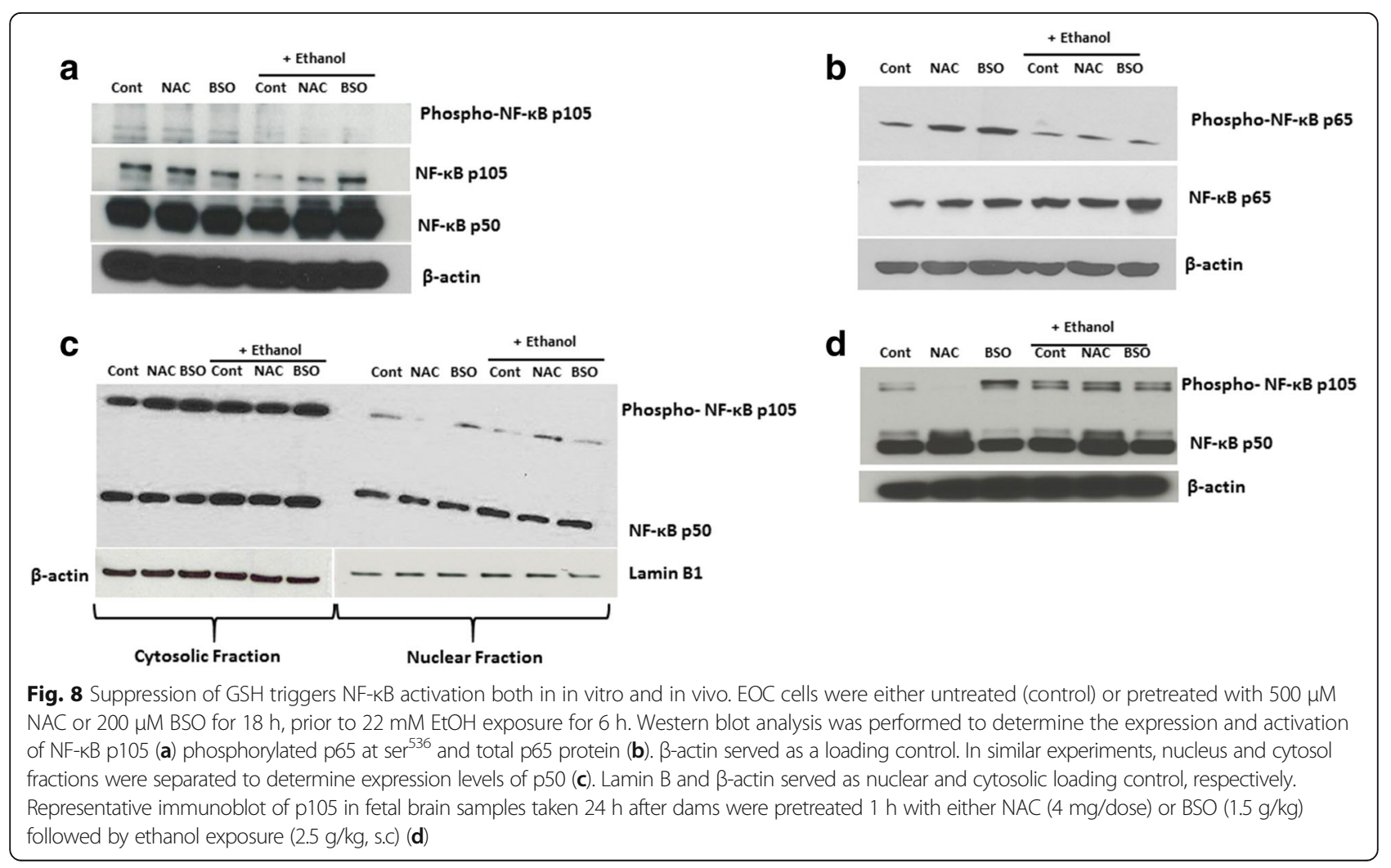


response (Figs. 3, 4, and 5). We emphasize again that GSH levels in the maternal brain remain unaltered across all treatment groups; interestingly however, ethanol, BSO, and NAC individually or in combination do elicit M1/M2 immune responses. This is always more striking in the fetal brain when compared to maternal brain (Figs. 3, 4, and 5). Inadequately developed fetal antioxidant system in early to mid-gestational stages is highly vulnerable to oxidative stress [55] and also explains the differential response seen between the maternal and the fetal brain.

Most distinctly, in the present study, we show a complex co-existence and alterations in microglia phenotype, which is primarily driven by GSH status. Notably, oxidative stress enhances the expression of pro-inflammatory cytokines IL- $1 \beta$, IFN- $\gamma$, and iNOS and simultaneously reduces anti-inflammatory cytokines IL-4 and IL-10, especially in groups exposed to $\mathrm{BSO}$ or $\mathrm{ETOH}$ alone or in combination (Figs. 3, 4, 6, and 7). These pro-inflammatory cytokines can directly or indirectly cause neuronal death, dysfunction, attenuate neurogenesis (our unpublished data), and impair spatial learning and memory function $[21,26,27]$. Conversely, treatment with NAC triggers the expression of M2-associated markers such as IL-10, IL-4, TGF- $\beta$, CCL22, and Arg and reduced the expression of M1-associated signals (IL-1 $\beta$, IFN- $\gamma$, and CCL9) (Figs. 3, 4, 6, and 7). Previous studies with adult rats found no changes in cytokine expression following ethanol exposure $[51,52]$ concur with our observations, wherein maternal brain tissues showed either a total absence of IL-10, and IFN- $\gamma$ mRNA levels or an insignificant difference of protein levels (IL-10,TNF- $\alpha$, IL1 $\beta, I F N-\gamma)$. Thus, a fully developed maternal antioxidant system is likely to resist transient fluctuations in oxidative stress and inflammation.

To determine the underlying mechanism of microglia activation and its phenotypic changes, we undertook in vitro experiments (Figs. 5, 6, 7, and 8). Exposure to ethanol and GSH depletion, alone or in combination, alters both the morphological and the functional characteristics of microglia cells by inducing ROS production (Figs. 5 and 6). Similar to our in vivo conditions, pretreatment with NAC maintained cellular GSH and, however, mitigated ethanolinduced ROS generation. In addition, ethanol and BSO exposure generate ROS (Fig. 5a, b) and deplete GSH levels (Fig. 5c). Ethanol augments ROS generation through the activation of NADPH oxidase (data not shown) [20]. GSH serves as a redox buffer against ROS [56], and its depletion is a distinctive feature of apoptotic cell death [57]. However, in this study, BSO treatment resulted in depleting cellular GSH pool and magnifying ROS production in microglia without affecting mitochondrial respiration rate and cell viability. This is likely driven in part by activation of $\mathrm{Nrf}-2$ pathways (Fig. 5d) as an adaptive cellular mechanism to counteract GSH perturbation by eliciting the expression of antioxidant genes, including $\mathrm{Cu} / \mathrm{Zn}$ SOD for ROS removal. A similar regulatory response to GSH depletion has been observed in other cellular models [58, 59]. In addition to aggravating oxidative damage, ROS also acts as a secondary messenger, modifying gene expression critical for survival [60].

Soluble factors in the microenvironment are known to regulate microglia polarization [28]. We found ROS and intracellular GSH to be critical for regulating cellular morphologies and a strong association exists between cell shape and inflammatory status of these cells (Figs. 6 and 7). Confocal data shows cells to predominantly assume round amoeboid shape under conditions of oxidative stress and redox imbalance. Conversely, microglia exhibit bipolar elongation when cellular redox homeostasis is maintained. Importantly, recent studies report the presence of similar bipolar/rod-shaped microglia at the site of injury, during the early phases of brain damage [61-63]. Such morphologies exert neuroprotective effects by producing greater amount of anti-inflammatory cytokines than pro-inflammatory cytokines [63]. Additionally, changes in morphology are closely associated with the functional activity of microglia [64, 65]. Our RT-PCR data shows that microglia with optimum GSH levels express significantly increased levels of IL-10 and CCL2 and subsequently downregulation of inflammatory cytokines and chemokines (IL-1 $\beta$, IL-6, and CCL9). On the other hand, depletion of cellular GSH results in microglia expressing higher levels of pro-inflammatory factors compared to control cells. Although ethanol induces microglia activation $[20,66]$, its polarizing potential on microglia has yet not been fully addressed. Our results establish a strong correlation between morphological alterations in microglia with gene expression profile distinctive to either M1- or M2-like phenotypes. The most wellcharacterized prototypic M2 marker is the expression of arginase1, and that of M1-like phenotype are IL-1 $\beta$ and iNOS [67-69]. Depletion of GSH resulted in a higher expression of M1-associated markers (IL-1 $\beta$ and iNOS) and exposure to NAC promoted Arg1 expression (Fig. 7 and Additional file 2: Figure S2). Together, these data demonstrate that intracellular GSH status influences cell morphology and modulates microglia polarization. Cell shape is reported to influence polarization in macrophages [34]. In agreement with this study, our results show that ethanol-induced morphological changes in microglia are highly attuned with cytokine and intensify its overall effect on M1 polarization state.

A mechanism that emerges from this study is that the plasticity and functional polarization of microglia is strongly influenced by oxidative stress [28], redox status, and pro- or anti-inflammatory factors [70]. 
Animals with perturbed redox homeostasis are prone to generate higher levels of ROS and acquire M1 phenotype. Acting as secondary messengers, ROS activate NF- $\mathrm{kB}$ signaling leading to transcription of proinflammatory cytokine genes. The present study revealed "non-classical" pathway for NF- $\mathrm{kB}$ activation. Western blot analysis of NF- $k B$ p105/50, phospho NF$\kappa B$ p105/50, NF- $\kappa B$ p65/RelA, and phospho NF- $\kappa B$ p65/Rel A reveals increased processing of p105 precursor protein via phosphorylation leading to overexpression of p50 and induction and phosphorylation of p65 in GSH depleted group which was further augmented by ethanol exposure. Of the various homo- and heterodimers of NF-kB, the p65/p50 dimer is the bestcharacterized inducer of pro-inflammatory genes and is fully functional in microglia [28]. On the other hand, p50 and p52 homodimers function as repressors due to their lack of a transcription activation domain [71]. Earlier studies have established p65/p50 as the primary mediator of NF- $\mathrm{kB}$ transcriptional activity for proinflammatory genes.
Expression of pro inflammatory cytokines and chemokines (IL-1 $\beta$, TNF- $\alpha$, IFN- $\gamma$, CCL9, CCL4, CCL3) subsequently incorporate microglia into the network of detrimental M1 phenotype. When abundant cellular GSH pools exist, M2-inducing signals such as IL-10 generally inhibit the expression of M1 chemokines. On the other hand, IL-10 inhibitory effects rely on both the inhibition of NF-kB [72] and STAT-dependent mechanism [73]. The schematic (Fig. 9) depicts the underlying mechanism of phenotypic acquisition of microglia under perturbed redox status and oxidative stress. We speculate that GSH content and ROS levels in microglia constitute a potential link for the crosstalk between Nrf-2 and NF-kB pathways, the two known crucial factor in regulating microglia dynamic and neuroinflammation [74], thereby driving the immunological phenotypic profiles.

\section{Conclusion}

In conclusion, our study shows that even a slight imbalance in the oxidative-redox homeostasis in the immature fetal

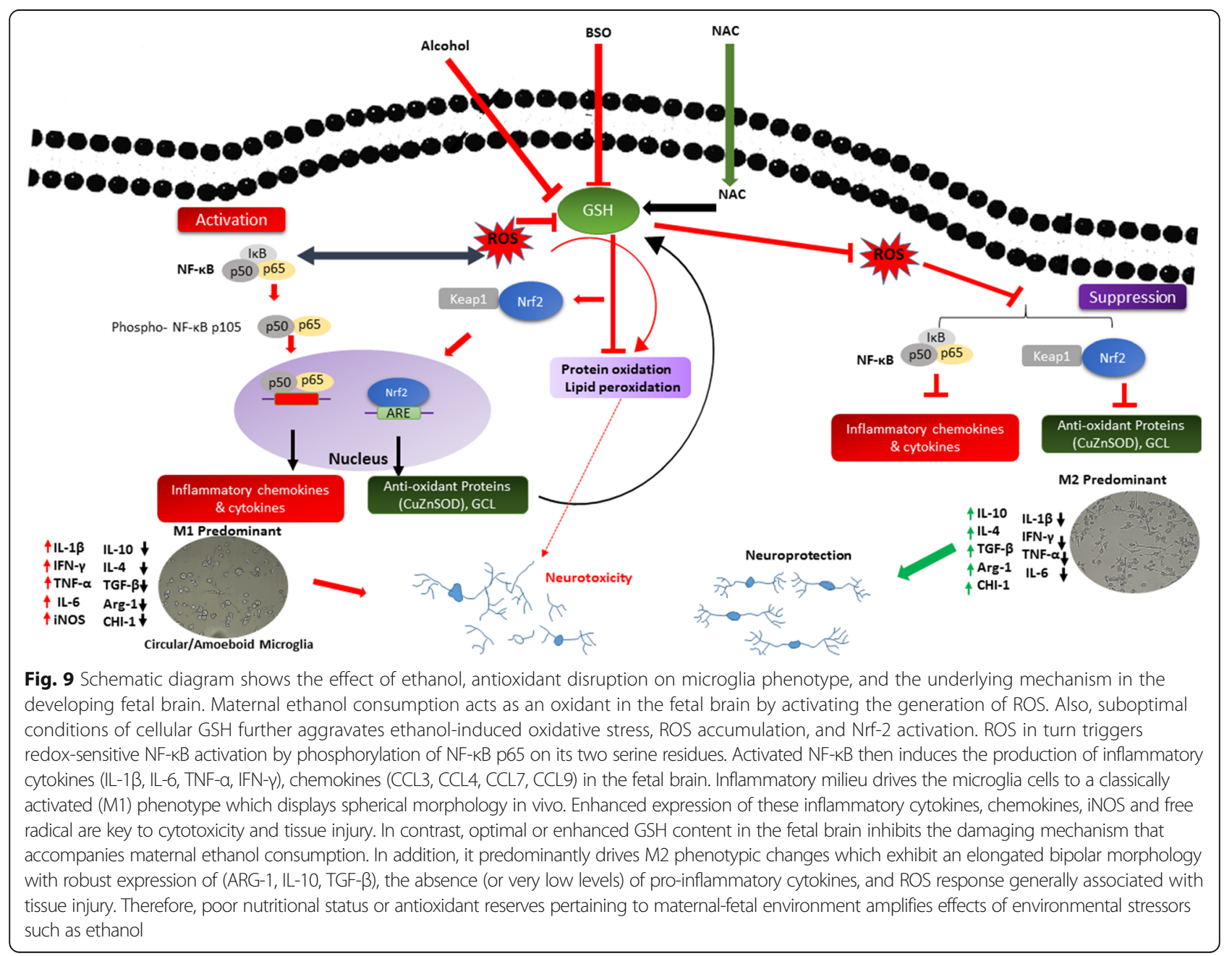


brain triggers activation of microglia towards a proinflammatory M1 phenotype that persists up to $24 \mathrm{~h}$. On the other hand, maintaining GSH levels triggers antiinflammatory M2-phenotypic response. Therefore, the existence of a bidirectional feedback loop between Nrf2 activation and phosphorylation of NFkB components p105 and $\mathrm{p} 65$, and their nuclear translocation associated with expression of inflammatory markers such as IL1- $\beta$ and IFN $-\gamma$, merits further exploration. Further, time-based studies will facilitate our understanding of the extent of fetal brain damage and whether these effects are reversible.

\section{Additional files}

Additional file 1: Figure S1. Concentration-dependent effect of ethano on cell viability. Plot of cell viability (MTS assay) obtained following $6 \mathrm{~h}$ treatment of cells with various concentration of ethanol. Data represent the average and standard deviation values from three replicate experiments. Where ${ }^{*} p \leq 0.05$. (TIFF $92 \mathrm{~kb}$ )

Additional file 2: Figure S2. Altered glutathione homeostasis impacts ethanol-induces superoxide dismutase expression in microglia. A representative Western blot of CUZnSOD expression in control and treatment groups. Actin served as the loading control. (TIFF $121 \mathrm{~kb}$ )

Additional file 3: Figure S3. Intracellular GSH is pivotal in EtOH-induced phenotypic acquisition of microglia. EOC 13.31 cells grown on coverslips were treated with NAC $(500 \mu \mathrm{M})$ or BSO $(200 \mu \mathrm{M})$ for $18 \mathrm{~h}$ prior to the presence or absence of EtOH (22 mM) exposure for $6 \mathrm{~h}$. Cells were labeled by antibodies against iNOS and Alexa Fluor 488 labeled secondary antibodies. The nuclei of the cells were counterstained with DAPI. GSH depletion by $\mathrm{BSO}$ and $\mathrm{EtOH}$ exposure synergistically exaggerate the expression of M1 marker (green) iNOS. Images were acquired on FV1000 confocal microscope equipped with a HeNe laser, 60x objective, NA 1.42 with an electronic zoom of 2. Scale bar $=40 \mu \mathrm{m}$ (TIFF $301 \mathrm{~kb})$

\section{Abbreviations}

Arg1: Arginase1; BSO: L-Buthionine sulfoximine; CCL2: Chemokine (C-C motif) ligand 2; CCL22: Chemokine (C-C motif) ligand 22; CCL3: Chemokine (C-C motif) ligand 3; CCL4: Chemokine (C-C motif) ligand 4; CCL-7: Chemokine (CC motif) ligand 7; CCL9: Chemokine (C-C motif) ligand 9; CCR2: C-C chemokine receptor type 2; Chi1: Chitinase 1; CUZnSOD: Superoxide dismutase; CXCL10: C-X-C motif chemokine 10; CXCR2: C-X-C chemokine receptor type 2; ELISA: Enzyme-linked immunosorbent assay; FASD: Fetal Alcohol Spectrum Disorder; GSH: Glutathione; HPLC: High-performance liquid chromatography; IFN Y: Interferon gamma; IL-10: Interleukin 10; IL13: Interleukin 1 beta; LL-4: Interleukin 4; IL-6: Interleukin 6; NAC: NAcetylcysteine; NFkB: Nuclear factor kappa-light-chain-enhancer of activated B cells; Nrf-2: Erythroid 2-related factor 2; OS: Oxidative stress; ROS: Reactive oxygen species; TGF- $\beta$ : Transforming growth factor beta 1; TLR: Toll like receptors

\section{Acknowledgements}

We thank Ms. Giovanina Hinojosa for technical assistance.

\section{Financial disclosure}

No authors have any financial interests relevant to this article to disclose.

\section{Funding}

This work was supported in part by funds from 59th Clinical Research Division, Lackland Air Force Base, TX, to CAR and from the RAHC-Edinburg to SKM. All fluorescent microscopy experiment images were generated in the Optical Imaging Core Facility at Edinburg - RAHC, Medical Research Division.

Availability of data and materials

Data are available on request to the authors.

\section{Authors' contributions}

FA carried out the studies and drafted the manuscript. KK and RA carried out the HPLC analysis of GSH. SLU was a graduate student in Reto Asmis's lab who ran the HPLC samples. GC and FA performed the statistical analysis. $S M$ and $C R$ conceived of the study and participated in its design and coordination and helped to draft the manuscript. All authors read and approved the final manuscript.

\section{Ethics approval}

This study was carried out in strict accordance with the recommendations in the Guide for the Care and Use of Laboratory Animals of the National Institutes of Health. All experiments were approved by the Institutional Animal Care and Use Committee of 59Th MDW Clinical Research Division, Lackland AFB, Texas.

\section{Consent for publication}

Not applicable.

\section{Competing interests}

The authors declare that they have no competing interests.

\section{Publisher's Note}

Springer Nature remains neutral with regard to jurisdictional claims in published maps and institutional affiliations.

\section{Author details}

${ }^{1}$ School of Medicine, Department of Biomedical Sciences, Regional Academic Health Center, University of Texas Rio Grande Valley, 1204 W. Schunior, Edinburg 78241, TX, USA. ${ }^{2}$ Department of Pediatrics, Uniformed Services University of Health Sciences \& Walter Reed National Military Medical Center, Jones Bridge Rd, Bethesda, MD, USA. ${ }^{3}$ Department of Medicine, The University of Texas Health Science Center, San Antonio, TX, USA. ${ }^{4}$ Department of Kinesiology, Health, and Nutrition, University of Texas at San Antonio, San Antonio, TX, USA. ${ }^{5}$ Department of Clinical Lab Sciences, The University of Texas Health Science Center, San Antonio, TX, USA.

${ }^{6}$ Department of Internal Medicine, University of Nebraska Health Science Center, Omaha, NE, USA.

Received: 8 May 2017 Accepted: 18 September 2017 Published online: 30 September 2017

\section{References}

1. Shim SY, Kim HS. Oxidative stress and the antioxidant enzyme system in the developing brain. Korean J Pediatr. 2013;56(3):107-11.

2. Williams JF, Smith VC, A. Committee On Substance. Fetal alcohol spectrum disorders. Pediatrics. 2015;136(5):e1395-406.

3. Bhang SY, et al. Maternal stress and depressive symptoms and infant development at six months: the Mothers and Children's Environmental Health (MOCEH) prospective study. J Korean Med Sci. 2016;31(6):843-51.

4. Knuesel I, et al. Maternal immune activation and abnormal brain development across CNS disorders. Nat Rev Neurol. 2014;10(11):643-60.

5. Cao M, et al. Fetal microglial phenotype in vitro carries memory of prior in vivo exposure to inflammation. Front Cell Neurosci. 2015;9:294.

6. Deverman BE, Patterson PH. Cytokines and CNS development. Neuron. 2009;64(1):61-78.

7. Thompson BL, Levitt P, Stanwood GD. Prenatal exposure to drugs: effects on brain development and implications for policy and education. Nat Rev Neurosci. 2009:10(4):303-12.

8. Ishii S, Hashimoto-Torii K. Impact of prenatal environmental stress on cortical development. Front Cell Neurosci. 2015;9:207.

9. Ikonomidou C, et al. Ethanol-induced apoptotic neurodegeneration and fetal alcohol syndrome. Science. 2000;287(5455):1056-60.

10. Maffi SK, et al. Glutathione content as a potential mediator of the vulnerability of cultured fetal cortical neurons to ethanol-induced apoptosis. J Neurosci Res. 2008:86(5):1064-76.

11. Guizzetti M, et al. Glia and neurodevelopment: focus on fetal alcohol spectrum disorders. Front Pediatr. 2014;2:123.

12. Topper $L A$, Baculis BC, Valenzuela CF. Exposure of neonatal rats to alcohol has differential effects on neuroinflammation and neuronal survival in the cerebellum and hippocampus. J Neuroinflammation. 2015;12:160. 
13. Henderson Gl, Chen JJ, Schenker S. Ethanol, oxidative stress, reactive aldehydes, and the fetus. Front Biosci. 1999;4:D541-50.

14. Cohen-Kerem R, Koren G. Antioxidants and fetal protection against ethanol teratogenicity. I. Review of the experimental data and implications to humans. Neurotoxicol Teratol. 2003;25(1):1-9.

15. Drew PD, Kane CJ. Fetal alcohol spectrum disorders and neuroimmune changes. Int Rev Neurobiol. 2014;118:41-80.

16. Kane CJ, Phelan KD, Drew PD. Neuroimmune mechanisms in fetal alcohol spectrum disorder. Dev Neurobiol. 2012;72(10):1302-16.

17. Kierdorf K, Prinz M. Factors regulating microglia activation. Front Cell Neurosci. 2013;7:44.

18. He J, Crews FT. Increased MCP-1 and microglia in various regions of the human alcoholic brain. Exp Neurol. 2008;210(2):349-58.

19. Qin L, Crews FT. Focal thalamic degeneration from ethanol and thiamine deficiency is associated with neuroimmune gene induction, microglial activation, and lack of monocarboxylic acid transporters. Alcohol Clin Exp Res. 2014;38(3):657-71.

20. Qin L, Crews FT. NADPH oxidase and reactive oxygen species contribute to alcohol-induced microglial activation and neurodegeneration. J Neuroinflammation. 2012;9:5.

21. Vink J, et al. Novel peptides prevent alcohol-induced spatial learning deficits and proinflammatory cytokine release in a mouse model of fetal alcohol syndrome. Am J Obstet Gynecol. 2005;193(3 Pt 1):825-9.

22. Tiwari V, Chopra K. Attenuation of oxidative stress, neuroinflammation, and apoptosis by curcumin prevents cognitive deficits in rats postnatally exposed to ethanol. Psychopharmacology. 2012;224(4):519-35.

23. Tiwari V, Chopra K. Resveratrol prevents alcohol-induced cognitive deficits and brain damage by blocking inflammatory signaling and cell death cascade in neonatal rat brain. J Neurochem. 2011;117(4):678-90.

24. Drew PD, et al. Pioglitazone blocks ethanol induction of microglial activation and immune responses in the hippocampus, cerebellum, and cerebral cortex in a mouse model of fetal alcohol spectrum disorders. Alcohol Clin Exp Res. 2015;39(3):445-54.

25. Michell-Robinson MA, et al. Roles of microglia in brain development, tissue maintenance and repair. Brain. 2015;138(Pt 5):1138-59.

26. Liao B, et al. Transformation from a neuroprotective to a neurotoxic microglial phenotype in a mouse model of ALS. Exp Neurol. 2012;237(1):147-52.

27. Kobayashi K, et al. Minocycline selectively inhibits M1 polarization of microglia. Cell Death Dis. 2013;4:e525.

28. Rojo Al, et al. Redox control of microglial function: molecular mechanisms and functional significance. Antioxid Redox Signal. 2014;21(12):1766-801.

29. Talalay P, Dinkova-Kostova AT, Holtzclaw WD. Importance of phase 2 gene regulation in protection against electrophile and reactive oxygen toxicity and carcinogenesis. Adv Enzym Regul. 2003;43:121-34.

30. Kharbanda KK, et al. Betaine treatment attenuates chronic ethanol-induced hepatic steatosis and alterations to the mitochondrial respiratory chain proteome. Int J Hepatol. 2012;2012:962183.

31. Asmis $R$, et al. A novel thiol oxidation-based mechanism for adriamycininduced cell injury in human macrophages. FASEB J. 2005;19(13):1866-8.

32. Zangarelli $A$, et al. Synergistic effects of caloric restriction with maintained protein intake on skeletal muscle performance in 21-month-old rats: a mitochondria-mediated pathway. FASEB J. 2006;20(14):2439-50.

33. Kamencic $\mathrm{H}$, et al. Monochlorobimane fluorometric method to measure tissue glutathione. Anal Biochem. 2000;286(1):35-7.

34. McWhorter FY, et al. Modulation of macrophage phenotype by cell shape. Proc Natl Acad Sci U S A. 2013;110(43):17253-8.

35. Orihuela R, McPherson CA, Harry GJ. Microglial M1/M2 polarization and metabolic states. Br J Pharmacol. 2016;173(4):649-65.

36. Potter ED, Ling ZD, Carvey PM. Cytokine-induced conversion of mesencephalicderived progenitor cells into dopamine neurons. Cell Tissue Res. 1999;296(2):235-46.

37. Gilmore $\mathrm{JH}$, et al. Prenatal infection and risk for schizophrenia: IL-1beta, IL-6, and TNFalpha inhibit cortical neuron dendrite development. Neuropsychopharmacology. 2004;29(7):1221-9.

38. Meyer $U$, et al. The time of prenatal immune challenge determines the specificity of inflammation-mediated brain and behavioral pathology. J Neurosci. 2006;26(18):4752-62.

39. Dziegielewska KM, et al. Acute-phase cytokines IL-1 beta and TNF-alpha in brain development. Cell Tissue Res. 2000;299(3):335-45.

40. Franklin $\mathrm{CL}$, Gruol DL. Acute ethanol alters the firing pattern and glutamate response of cerebellar Purkinje neurons in culture. Brain Res. 1987:416(2):205-18
41. Qin ZH, Tao LY, Chen X. Dual roles of NF-kappaB in cell survival and implications of NF-kappaB inhibitors in neuroprotective therapy. Acta Pharmacol Sin. 2007;28(12):1859-72.

42. Imielski $Y$, et al. Regrowing the adult brain: NF-kappaB controls functional circuit formation and tissue homeostasis in the dentate gyrus. PLoS One. 2012;7(2):e30838.

43. Kaltschmidt B, Kaltschmidt C. NF-kappaB in the nervous system. Cold Spring Harb Perspect Biol. 2009;1(3):a001271.

44. Hill AJ, et al. The role of NADPH oxidase in a mouse model of fetal alcohol syndrome. Am J Obstet Gynecol. 2014;210(5):466 e1-5.

45. Keen $\mathrm{CL}$, et al. The plausibility of maternal nutritional status being a contributing factor to the risk for fetal alcohol spectrum disorders: the potential influence of zinc status as an example. Biofactors. 2010;36(2):125-35.

46. Henderson $\mathrm{Gl}$, et al. In utero ethanol exposure elicits oxidative stress in the rat fetus. Alcohol Clin Exp Res. 1995;19(3):714-20.

47. Brolese $\mathrm{G}$, et al. Pre- and postnatal exposure to moderate levels of ethanol can have long-lasting effects on hippocampal glutamate uptake in adolescent offspring. PLoS One. 2015;10(5):e0127845.

48. Lante $\mathrm{F}$, et al. Late $\mathrm{N}$-acetylcysteine treatment prevents the deficits induced in the offspring of dams exposed to an immune stress during gestation. Hippocampus. 2008;18(6):602-9.

49. Merlot E, Couret D, Otten W. Prenatal stress, fetal imprinting and immunity. Brain Behav Immun. 2008;22(1):42-51.

50. Kane CJ, et al. Protection of neurons and microglia against ethanol in a mouse model of fetal alcohol spectrum disorders by peroxisome proliferator-activated receptor-gamma agonists. Brain Behav Immun. 2011; 25(Suppl 1):S137-45

51. McClain JA, et al. Adolescent binge alcohol exposure induces long-lasting partial activation of microglia. Brain Behav Immun. 2011;25(Suppl 1):S120-8.

52. Marshall SA, et al. Microglial activation is not equivalent to neuroinflammation in alcohol-induced neurodegeneration: The importance of microglia phenotype. Neurobiol Dis. 2013;54:239-51.

53. Pratt $L$, et al. Maternal inflammation promotes fetal microglial activation and increased cholinergic expression in the fetal basal forebrain: role of interleukin-6. Pediatr Res. 2013;74(4):393-401.

54. Prinz M, Priller J. Microglia and brain macrophages in the molecular age: from origin to neuropsychiatric disease. Nat Rev Neurosci. 2014; 15(5):300-12.

55. Sheldon RA, et al. Manipulation of antioxidant pathways in neonatal murine brain. Pediatr Res. 2004;56(4):656-62.

56. Song J, et al. Glutathione protects brain endothelial cells from hydrogen peroxide-induced oxidative stress by increasing nrf2 expression. Exp Neurobiol. 2014;23(1):93-103.

57. Franco R, Cidlowski JA. Apoptosis and glutathione: beyond an antioxidant. Cell Death Differ. 2009;16(10):1303-14.

58. Filomeni $\mathrm{G}$, et al. Antiapoptotic response to induced GSH depletion: involvement of heat shock proteins and NF-kappaB activation. Antioxid Redox Signal. 2005;7(3-4):446-55.

59. Lee HR, et al. Adaptive response to GSH depletion and resistance to Lbuthionine-(S,R)-sulfoximine: involvement of Nrf2 activation. Mol Cell Biochem. 2008;318(1-2):23-31.

60. Block ML, Zecca L, Hong JS. Microglia-mediated neurotoxicity: uncovering the molecular mechanisms. Nat Rev Neurosci. 2007:8(1):57-69.

61. Taylor SE, et al. Rod microglia: a morphological definition. PLoS One. 2014; 9(5):e97096.

62. Ziebell JM, et al. Rod microglia: elongation, alignment, and coupling to form trains across the somatosensory cortex after experimental diffuse brain injury. J Neuroinflammation. 2012;9:247.

63. Tam WY, Ma CH. Bipolar/rod-shaped microglia are proliferating microglia with distinct M1/M2 phenotypes. Sci Rep. 2014;4:7279.

64. Szabo M, Gulya K. Development of the microglial phenotype in culture. Neuroscience. 2013;241:280-95.

65. Stence N, Waite M, Dailey ME. Dynamics of microglial activation: a confocal time-lapse analysis in hippocampal slices. Glia. 2001;33(3):256-66.

66. Qin $\mathrm{L}$, et al. Increased systemic and brain cytokine production and neuroinflammation by endotoxin following ethanol treatment. J Neuroinflammation. 2008:5:10.

67. Yao A, et al. Programmed death 1 deficiency induces the polarization of macrophages/microglia to the M1 phenotype after spinal cord injury in mice. Neurotherapeutics. 2014;11(3):636-50. 
68. Hernandez-Rabaza V, et al. Neuroinflammation increases GABAergic tone and impairs cognitive and motor function in hyperammonemia by increasing GAT-3 membrane expression. Reversal by sulforaphane by promoting M2 polarization of microglia. J Neuroinflammation. 2016;13(1):83.

69. Tang Y, Le W. Differential Roles of M1 and M2 Microglia in Neurodegenerative Diseases. Mol Neurobiol. 2016;53(2):1181-94.

70. Mantovani A, et al. The chemokine system in diverse forms of macrophage activation and polarization. Trends Immunol. 2004;25(12):677-86.

71. Porta $C$, et al. Tolerance and M2 (alternative) macrophage polarization are related processes orchestrated by p50 nuclear factor kappaB. Proc Natl Acad Sci U S A. 2009:106(35):14978-83.

72. Li Q, Verma IM. NF-kappaB regulation in the immune system. Nat Rev Immunol. 2002;2(10):725-34.

73. Lang $R$, et al. Shaping gene expression in activated and resting primary macrophages by IL-10. J Immunol. 2002;169(5):2253-63.

74. Rojo Al, et al. Nrf2 regulates microglial dynamics and neuroinflammation in experimental Parkinson's disease. Glia. 2010;58(5):588-98.

Submit your next manuscript to BioMed Central and we will help you at every step:

- We accept pre-submission inquiries

- Our selector tool helps you to find the most relevant journal

- We provide round the clock customer support

- Convenient online submission

- Thorough peer review

- Inclusion in PubMed and all major indexing services

- Maximum visibility for your research

Submit your manuscript at www.biomedcentral.com/submit
Biomed Central 